\title{
Geant4 Simulation Study of Deep Underground Muons: Vertical Intensity and Angular Distribution
}

\author{
Halil Arslan and Mehmet Bektasoglu \\ Department of Physics, Sakarya University, 54187 Sakarya, Turkey \\ Correspondence should be addressed to Mehmet Bektasoglu; mehmetb@sakarya.edu.tr
}

Received 28 July 2013; Accepted 19 September 2013

Academic Editor: Carlo Cattani

Copyright (C) 2013 H. Arslan and M. Bektasoglu. This is an open access article distributed under the Creative Commons Attribution License, which permits unrestricted use, distribution, and reproduction in any medium, provided the original work is properly cited.

\begin{abstract}
Underground muon intensities up to 10000 m.w.e. and angular distribution up to 6500 m.w.e. in standard rock have been investigated using Geant 4 simulation package. Muons with energies above $100 \mathrm{GeV}$ were distributed from the ground level taking into account the muon charge ratio of $\sim 1.3$ at sea level. The simulated differential muon intensities are in good agreement with the intensities given in the literature. Furthermore, the simulation results for the integrated intensities are consistent with the experimental data, particularly at depths above 4000 m.w.e., where the simulation gives slightly smaller intensities than the experimental ones. In addition, the simulated exponent $n$ at different underground depths agrees well with the experimental points, especially above $\sim 2000$ m.w.e.
\end{abstract}

\section{Introduction}

Interaction of the primary cosmic rays (mostly protons and alpha particles) with the atmospheric nuclei produces secondary cosmic rays that are able to reach the Earth's surface. Among the mentioned secondary cosmic rays are muons, which are the most abundant charged particles at sea level. They interact weakly with the media they propagate in and can even penetrate large thicknesses of water, ice, or rock. The energy loss mechanisms, such as ionization and atomic excitation, bremsstrahlung, electron pair production, and photonuclear interactions, play a role while muons propagate in the matter [1].

Significant pieces of information can be achieved from underground or underwater muon investigations. For instance, a direct correlation exists between underground muon intensities and meson production in the stratosphere [2] due to different sensitivities of pions and kaons against temperature changes. Furthermore, deep underground muon data are useful for background estimations in the underground areas housing neutrino experiments, and muon intensity measurements at various depths yield information on the electromagnetic processes that reduce the flux [1]. Additionally, information on the spectrum, as well as the composition, of the primary cosmic rays with energies above $\sim 10^{14} \mathrm{eV}$ can be obtained from deep underground muon data. Therefore, underground muon studies have been attracting a continuous attention for the past several decades (see, for instance, [3-10]). Recently, Monte Carlo simulations have also been utilized in order to investigate intensity [11] and angular distributions [12] of muons at relatively shallower depths.

As the high energy muons are not affected by the magnetic field of the Earth, they show no azimuthal dependence at sea level. Since only the energetic muons reach significant depths, underground muons have no azimuthal dependence as well. On the other hand, underground muons do show a zenithal dependence. This is because muons with larger zenith angles, compared to the ones with smaller angles, lose more energy in the medium they propagate through. Therefore, the intensity of muons incident with a large zenith angle reaching a particular depth is expected to be smaller than that of vertical muons. The zenith angle distribution of underground muons could be expressed by

$$
I(\theta, h)=I\left(0^{\circ}, h\right) \cos ^{n}(\theta),
$$

where $\theta$ is the zenith angle, $I\left(0^{\circ}, h\right)$ is the vertical intensity, and $n$ is an exponent at the depth of $h$. The exponent $n$, which 
works for zenith angles $\theta \leq 40^{\circ}$ and depths up to $\sim 5000$ m.w.e. [4], is given by

$$
n=1.53+8.0 \cdot 10^{-4} h+\epsilon,
$$

where the depth $h$ is in $\mathrm{hg} / \mathrm{cm}^{2}$ (m.w.e.) and $\epsilon$ is a small correction coming from muon decay and ionization losses at shallower depths [1]. Since at greater slant depths the neutrino induced muons dominate, the usage of the cosine power low of angular distribution becomes inadequate.

In the present study, deep underground muon intensity up to 10000 m.w.e. and angular distribution up to 6500 m.w.e. in standard rock have been investigated using Monte Carlo simulations, and the simulation results have been compared with measurements performed by different groups. The limit of 10000 m.w.e. was selected due to the reason that for deeper depths neutrino-induced muons start to contribute to the muon intensity dominantly.

\section{Simulation}

Geant4 (for GEometry ANd Tracking) is an object-oriented toolkit, simulating the passage of particles through matter [14] using Monte Carlo methods. This simulation package is being intensively used in several areas including nuclear and particle physics, medical and space physics. Its usage for the studies of various cosmic muon properties, both at sea level [15-18] and underground [11,12], has recently grown.

In this work, deep underground vertical intensity and angular distribution of cosmic muons are investigated using Geant4 simulation package (release 4.9.3.p01). The Earth's crust was modeled as a rectangular box made of standard rock, which has been defined as a mixture of $\mathrm{CaCO}_{3}$ and $\mathrm{MgCO}_{3}$ with an average density of $\rho=2.65 \mathrm{~g} / \mathrm{cm}^{3}, Z / A=$ 0.50 , and $Z^{2} / A=5.50$ ( $Z$ and $A$ being the atomic and mass number, resp.) [19].

Positively and negatively charged muons with energies above $100 \mathrm{GeV}$ were distributed isotropically [18] $\left(\theta \leq 60^{\circ}\right)$ from the ground level taking into account the muon charge ratio of $\sim 1.3$ at sea level (see, e.g., [20]). The threshold energy of $100 \mathrm{GeV}$ is selected in order to eliminate the low energy muons that do not have enough energy to reach the underground depth of interest. Energy distribution of the primary muons used as input in the simulations was resembled to the measurements by Rastin [13] below $3 \mathrm{TeV}$. For muons with energies high enough to reach deep underground, the distribution was extrapolated up to $20 \mathrm{TeV}$ (see Figure 1).

The Low and High Energy Parameterization (LHEP) and the standard electromagnetic (emstandard) models have been used for hadronic and electromagnetic interactions, respectively. Detailed information on these models can be found in the Geant4 web page [21].

Energies and zenith angles of the muons reaching the depth up to 10000 m.w.e. in the simulated rock sample were recorded for 20 levels with 500 m.w.e. increment. The integrated intensity for each level up to 10000 m.w.e. and the angular distribution up to 6500 m.w.e. were determined. The following section discusses the results.

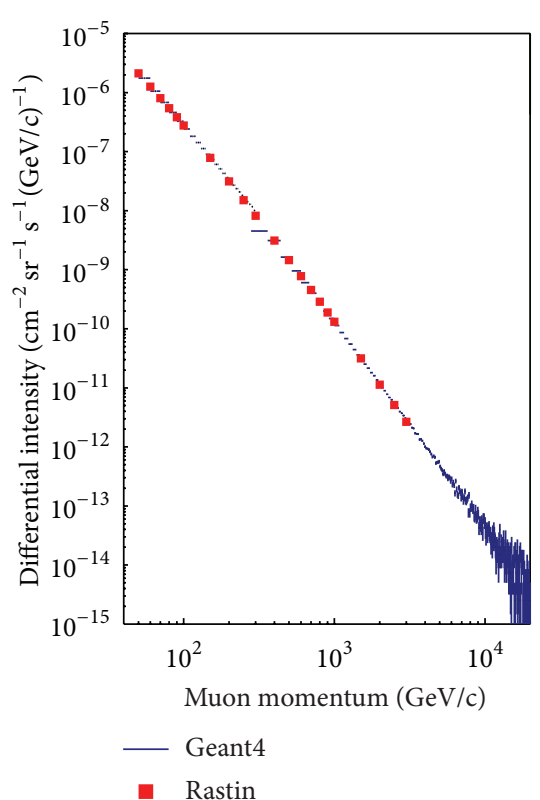

FIGURE 1: Sea level muon distribution: measurements by Rastin [13] and primary muons used in Geant 4 simulation (color online).

\section{Results and Discussion}

The simulation results for the normalized local spectrum, which was defined as the differential energy spectrum normalized to the corresponding integrated intensity, of underground muons are given in Figure 2 for various depths in standard rock. The figure, in which the error bars are smaller than the marker size for most of the bins, illustrates that for relatively low muon energies (up to $\sim 100 \mathrm{GeV}$ ) the spectrum has a tendency to become flat with increasing depth. For the depth $h>2500$ m.w.e., the underground differential spectrum of muons is again constant for low muon energies but, independent of the depth, steepens to reflect the surface muon spectrum at muon energies above $\sim 500 \mathrm{GeV}$. One can also notice that the shape of the spectrum also seems to become independent of depth as the depth gets bigger than $h>2500$ m.w.e. These results are in good agreement with the information in the literature $[22,23]$.

The integral intensity of muons underground obtained from the Geant 4 simulation is illustrated in Figure 3 as a function of depth in the range 500-10000 m.w.e. with 500 m.w.e. increment. Also in the figure are shown several data points from various experiments $[3,5,8]$. One may conclude that the simulation reproduces the experimental results well especially at depths above 4000 m.w.e, below which the simulation yields slightly smaller intensities than those the experiments give. The red solid line indicates the fit made to the simulation results using the Fréjus function [24]:

$$
I_{\mu}(h)=A\left(\frac{h_{0}}{h}\right)^{2} e^{-h / h_{0}} .
$$

The fit yields $A=(0.89 \pm 0.07) \times 10^{-6} \mathrm{~cm}^{-2} \mathrm{~s}^{-1} \mathrm{sr}^{-1}$ and $h_{0}=1307 \pm 3$ m.w.e. The fit parameters obtained from 


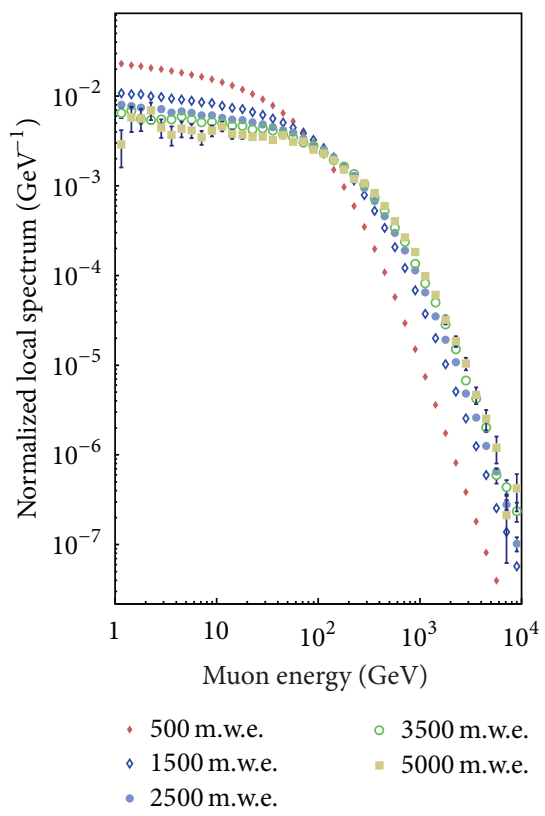

FIgURE 2: Normalized differential muon intensities in selected depths of standard rock (color online).

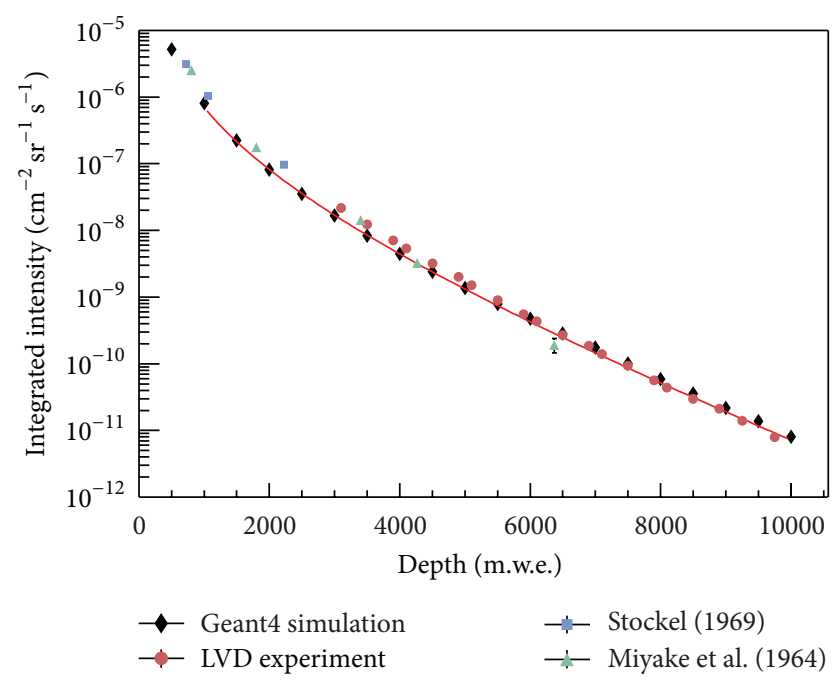

FIGURE 3: Integrated intensity of muons as a function of depth (color online).

TABLE 1: Fit parameters from intensity-depth relation in (3).

\begin{tabular}{lcc}
\hline Experiment & $A\left(\times 10^{-6} \mathrm{~cm}^{-2} \mathrm{~s}^{-1} \mathrm{sr}^{-1}\right)$ & $h_{0}$ (m.w.e. $)$ \\
\hline Fréjus 1989 & $1.96 \pm 0.09$ & $1184 \pm 8$ \\
LVD 1995 & $1.77 \pm 0.02$ & $1211 \pm 3$ \\
Fréjus 1996 & $2.18 \pm 0.05$ & $1127 \pm 4$ \\
MACRO 1995 & $1.81 \pm 0.06$ & $1231 \pm 1$ \\
\hline Geant4 & $0.89 \pm 0.07$ & $1307 \pm 3$ \\
\hline
\end{tabular}

various experiments (Fréjus 1989 [24], LVD 1995 [25], Fréjus 1996 [26], and MACRO 1995 [7]) together with the ones from the present work are given in Table 1 .

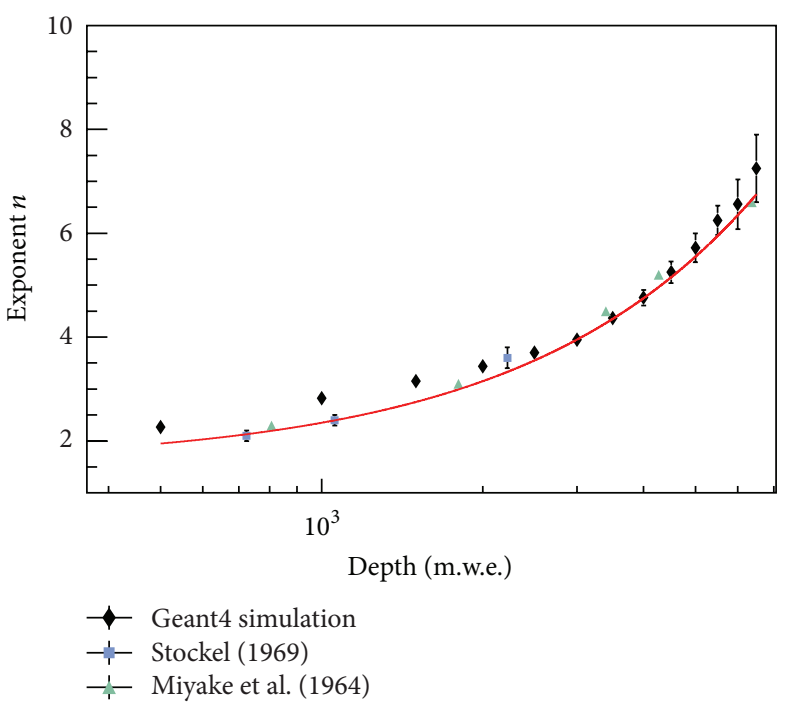

FIgURE 4: The simulated and experimental exponent $n$ as a function of depth in standard rock (color online).

Lastly, the exponent $n$ has been extracted for every 500 m.w.e., starting from 500 m.w.e. up to 6500 m.w.e. depth, by fitting the simulated zenith angular distribution of underground muons with the expression $I(\theta)=I\left(0^{\circ}\right) \cos ^{n}(\theta)$ for $\theta \leq 40^{\circ}$. The obtained values of $n$ have been drawn as a function of depth in standard rock (see Figure 4). The figure also includes the exponent values from two experiments $[3,5]$. Comparison between the exponent values obtained from the simulation with the ones obtained from the measurements shows that the simulation gives very consistent results with the experimental ones, especially above $\sim 2000$ m.w.e. Below that depth, the simulation yields slightly larger exponent values. The red solid line represents the function given in (2).

\section{Conclusions}

In the present simulation study, underground muon intensity up to $10000 \mathrm{~m}$.w.e. and angular distribution up to 6500 m.w.e. in standard rock have been investigated using Geant 4 simulation package. The differential muon intensities obtained from the simulation are in good agreement with the ones in the literature. Also, the simulation results for the integrated intensities are consistent with the experimental data especially at depths above 4000 m.w.e., below which the simulation yields slightly smaller intensities than the experiments. Furthermore, the exponent $n$ for each depth underground agrees, in general, well with the experimental points. Slight disagreement between the simulated and experimental $n$ values for depths below $\sim 2000$ m.w.e. could be attributed to the interaction models and is the subject of further investigations.

\section{References}

[1] P. K. F. Grieder, Cosmic Rays at Earth: Researcher's Reference Manual and Data Book, Elsevier, Amsterdam, The Netherlands, 2001. 
[2] E. W. Grashorn, J. K. de Jong, M. C. Goodman et al., "The atmospheric charged kaon/pion ratio using seasonal variation methods," Astroparticle Physics, vol. 33, no. 3, pp. 140-145, 2010.

[3] S. Miyake, V. S. Narasimham, and P. V. Ramana Murthy, "Cosmic-ray intensity measurements deep undergound at depths of (800-8400) m w.e.," Il Nuovo Cimento, vol. 32, no. 6, pp. 1505-1523, 1964.

[4] M. G. K. Menon, S. Naranan, V. S. Narasimham et al., "Muon intensities and angular distributions deep underground," Proceedings of the Physical Society, vol. 90, no. 3, pp. 649-656, 1967.

[5] C. T. Stockel, "A study of muons deep underground. I. Angular distribution and vertical intensity," Journal of Physics A, vol. 2, no. 6, pp. 639-649, 1969.

[6] J. N. Crookes and B. C. Rastin, "The absolute intensity of muons at $31.6 \mathrm{hg} \mathrm{cm}^{-2}$ below sea-level," Nuclear Physics B, vol. 58, no. 1, pp. 93-109, 1973.

[7] M. Ambrosio, R. Antolini, G. Auriemma et al., "Vertical muon intensity measured with MACRO at the Gran Sasso laboratory," Physical Review D, vol. 52, no. 7, pp. 3793-3802, 1995.

[8] M. Aglietta, B. Alpat, E. D. Alyea et al., "Muon "depth-intensity" relation measured by the LVD underground experiment and cosmic-ray muon spectrum at sea level," Physical Review D, vol. 58, no. 9, Article ID 092005, 6 pages, 1998.

[9] P. Adamson, C. Andreopoulos, D. J. Auty et al., "Measurement of the underground atmospheric muon charge ratio using the MINOS near detector," Physical Review D, vol. 83, no. 3, Article ID 032011, 10 pages, 2011.

[10] B. Mitrica, "Design study of an underground detector for measurements of the differential muon flux," Advances in High Energy Physics. In press.

[11] M. Bektasoglu, H. Arslan, and D. Stanca, "Simulations of muon flux in slanic salt mine," Advances in High Energy Physics, vol. 2012, Article ID 751762, 8 pages, 2012.

[12] M. Bektasoglu and H. Arslan, "Simulation of the zenith angle dependence of cosmic muon intensity in Slanic salt mine with Geant4," Romanian Journal of Physics, vol. 59, no. 1-2, 2014.

[13] B. C. Rastin, "An accurate measurement of the sea-level muon spectrum within the range 4 to $3000 \mathrm{GeV} / \mathrm{c}$," Journal of Physics $G$, vol. 10, no. 11, pp. 1609-1628, 1984.

[14] S. Agostinelli, J. Allison, K. Amako et al., "Geant4-a simulation toolkit," Nuclear Instruments and Methods in Physics Research A, vol. 506, no. 3, pp. 250-303, 2003.

[15] M. Bektasoglu and H. Arslan, "Estimation of the effects of the Earth's electric and magnetic fields on cosmic muons at sea level by Geant4," Journal of Atmospheric and Solar-Terrestrial Physics, vol. 74, pp. 212-216, 2012.

[16] H. Arslan and M. Bektasoglu, "Azimuthal angular dependence study of the atmospheric muon charge ratio at sea level using Geant4," Journal of Physics G, vol. 39, no. 5, Article ID 055201, 2012.

[17] M. Bektasoglu and H. Arslan, "Investigation of the zenith angle dependence of cosmic-ray muons at sea level," Pramana, vol. 80, no. 5, pp. 837-846, 2013.

[18] H. Arslan and M. Bektasoglu, "Geant4 simulation for the zenith angle dependence of cosmic muon intensities at two different geomagnetic locations," International Journal of Modern Physics A, vol. 28, no. 16, Article ID 1350071, 11 pages, 2013.

[19] J. Reichenbacher and J. de Jong, "Calculation of the underground muon inensity crouch curve from a parameterization of the flux at surface," in Proceeding of the 30th International Cosmic Ray Conference, Mexico City, Mexico, 2008.
[20] S. Haino, T. Sanuki, K. Abe et al., "Measurements of primary and atmospheric cosmic-ray spectra with the BESS-TeV spectrometer," Physics Letters B, vol. 594, no. 1-2, pp. 35-46, 2004.

[21] Geant4, 2013, http://geant4.cern.ch/support/.

[22] T. Gaisser, Cosmic Rays and Particle Physics, Cambridge University Press, Cambridge, UK, 1990.

[23] G. L. Cassiday, J. W. Keuffel, and J. A. Thompson, "Calculation of the stopping-muon rate underground," Physical Review D, vol. 7, no. 7, pp. 2022-2031, 1973.

[24] Ch. Berger, M. Fröhlich, H. Mönch et al., "Experimental study of muon bundles observed in the Fréjus detector," Physical Review D, vol. 40, no. 7, pp. 2163-2171, 1989.

[25] M. Aglietta, B. Alpat, E. D. Alyea et al., "Neutrino-induced and atmospheric single-muon fluxes measured over five decades of intensity by LVD at Gran Sasso Laboratory," Astroparticle Physics, vol. 3, no. 4, pp. 311-320, 1995.

[26] W. Rhode, K. Daum, P. Bareyre et al., "Limits on the flux of very high energy neutrinos with the Fréjus detector," Astroparticle Physics, vol. 4, no. 3, pp. 217-225, 1996. 

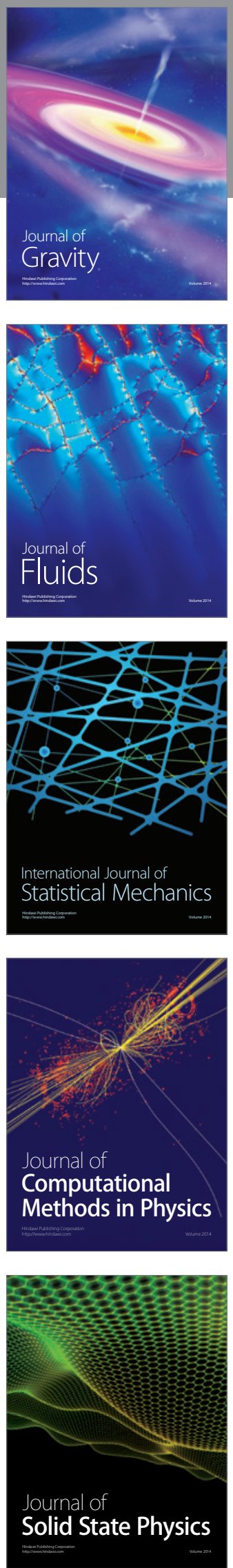

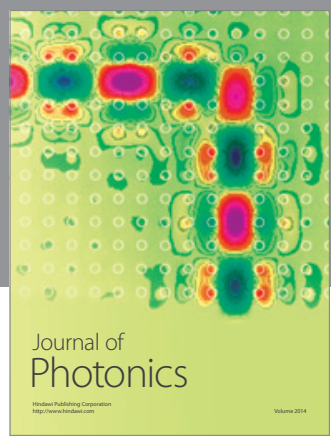

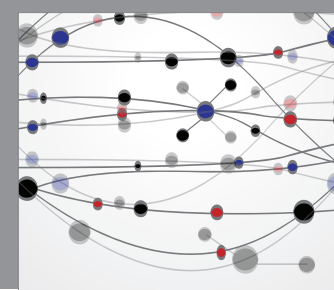

The Scientific World Journal

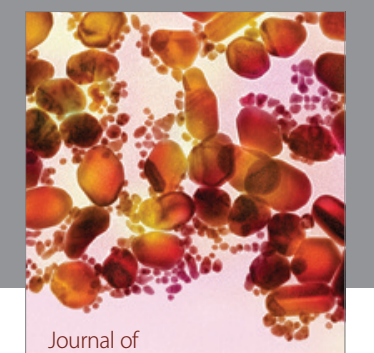

Soft Matter
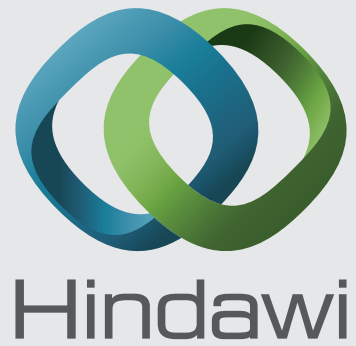

Submit your manuscripts at

http://www.hindawi.com
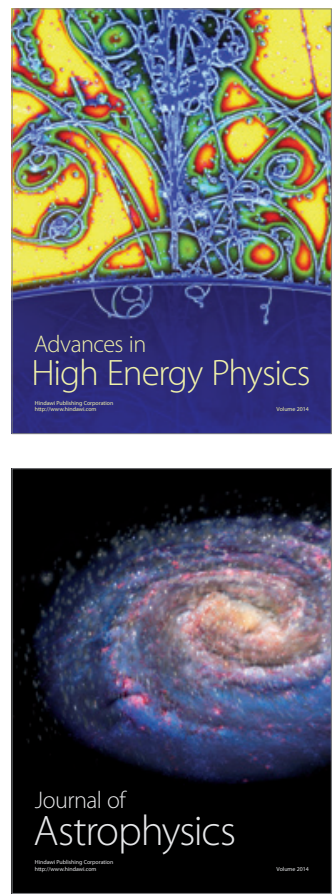
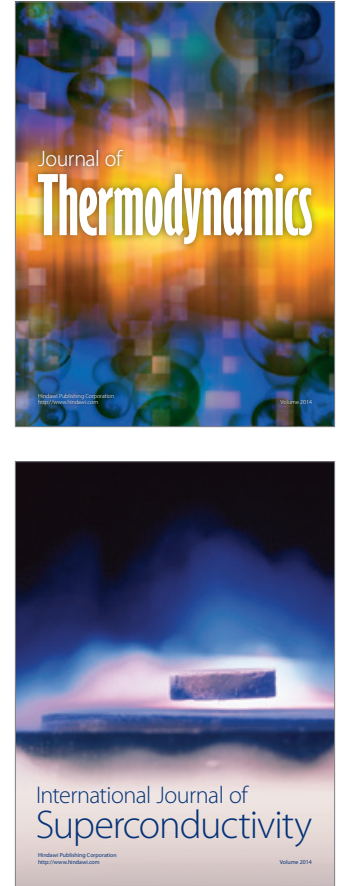
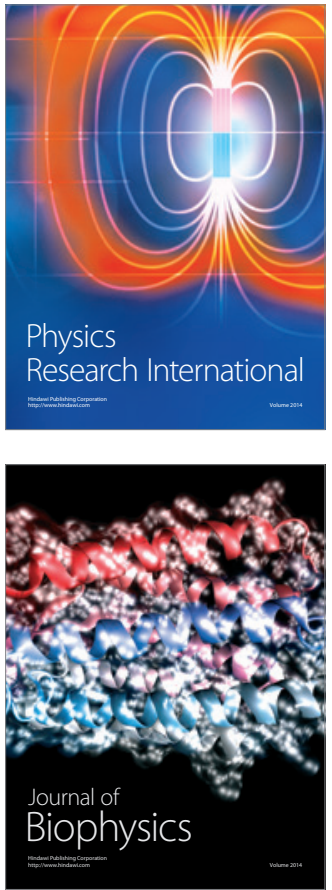
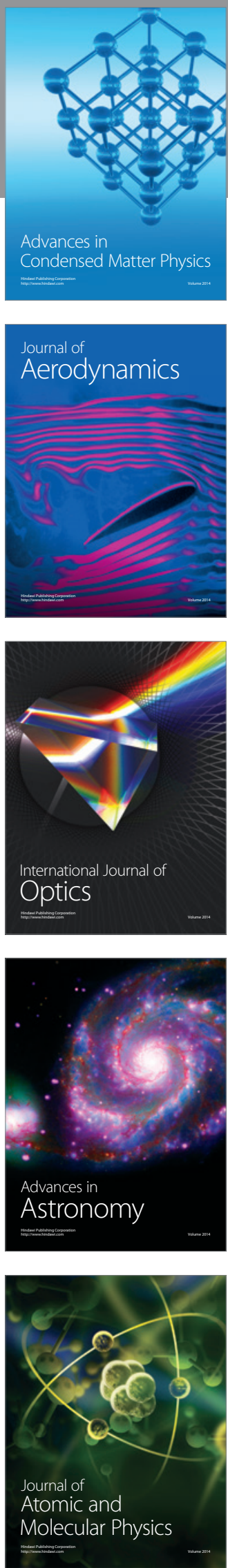